\title{
«Pour acquerir honneur et pris». Textes réunis et publiés par Maria Colombo Timelli et Claudio Galderisi
}

\section{Paola Cifarelli}

\section{(2) OpenEdition}

Journals

\section{Edizione digitale}

URL: http://journals.openedition.org/studifrancesi/29987

DOI: $10.4000 /$ studifrancesi.29987

ISSN: 2421-5856

\section{Editore}

Rosenberg \& Sellier

\section{Edizione cartacea}

Data di pubblicazione: 1 avril 2006

Paginazione: 131-134

ISSN: 0039-2944

\section{Notizia bibliografica digitale}

Paola Cifarelli, ««Pour acquerir honneur et pris». Textes réunis et publiés par Maria Colombo Timelli et Claudio Galderisi», Studi Francesi [Online], 148 (XLX | I) | 2006, online dal 30 novembre 2015, consultato il 19 avril 2021. URL: http://journals.openedition.org/studifrancesi/29987 ; DOI: https://doi.org/

10.4000/studifrancesi.29987

Questo documento è stato generato automaticamente il 19 avril 2021.

\section{cc) (†) $\odot$}

Studi Francesi è distribuita con Licenza Creative Commons Attribuzione - Non commerciale - Non opere derivate 4.0 Internazionale. 


\title{
«Pour acquerir honneur et pris». Textes réunis et publiés par Maria Colombo Timelli et Claudio Galderisi
}

\author{
Paola Cifarelli
}

\section{NOTIZIA}

«Pour acquerir honneur et pris». Mélanges de Moyen Français offerts à Giuseppe Di Stefano. Textes réunis et publiés par MARIA COLOMBO TIMELLI et CLAUDIO GALDERISI, Montréal, CERES, 2004, pp. 625.

1 Questa imponente miscellanea, cui hanno contribuito i più eminenti specialisti del moyen français, rende il giusto omaggio ad uno degli studiosi che, negli ultimi decenni, hanno impresso un impulso vigoroso alla ricerca in quest'ambito. I suoi fondamentali lavori nel campo della lingua e della letteratura di questo periodo hanno aperto il cammino verso nuovi e stimolanti campi di indagine, mentre la sua intensa attività come direttore della rivista «Le Moyen Français» e di varie collane editoriali, ma anche come organizzatore di convegni e didatta, ha creato occasioni fruttuose per lo scambio del sapere e la diffusione delle conoscenze.

2 Maria Colombo Timelli e Claudio Galderisi, curatori del volume ed autori di un'introduzione che ripercorre le tappe fondamentali dell'attività scientifica di Giuseppe Di Stefano, hanno saputo orientare ed organizzare i numerosissimi contributi degli studiosi secondo gli assi principali che sottendono la vasta produzione scientifica dell'illustre studioso. La raccolta si apre dunque con una sezione dedicata alla Littérature parénétique, costituita da quattro studi; quello di MAUREen BOULTON ('les Histoires de la Bible' en anglo-normand: une Bible factice, pp. 17-26) riguarda la tradizione manoscritta e l'analisi delle fonti di un adattamento biblico anglo-normanno trecentesco, realizzato a partire da fonti molto eterogenee e con un intento didattico che guida la strutturazione originale della materia biblica. LESLIE C. BROOKS (Le monde 
corrompu: Le 'Songe de Pestilence', pp. 27-35) analizza l'opera in prosa trecentesca attribuita a Henri des Ferrières che costituisce la seconda parte dei Livres du Roy Modus et de la Royne Ratio, sottolineandone l'intento moralizzante. ALAIN CORBELLARI (Jacques de Bugnin entre deux mondes, pp. 37-45) rivaluta almeno in parte le caratteristiche formali del Congié pris du siècle séculier, composto da Jacques de Bugnin verso il 1480 e giudicato severamente da A. Piaget, che fornì l'edizione di questo poema in cui si coniugano semplicità del dispositivo e raffinatezze retoriche. CINZIA PIGNATELLI (Un traducteur qui affiche ses croyances: l'ajout d'exempla au corpus des 'Otia Imperialia' de Gervais de Tilbury dans la traduction attribuée à Jean d'Antioche, pp. 47-54) analizza quattro racconti introdotti autonomamente dal traduttore al testo latino degli Otia Imperialia e mostra che essi concorrono in modo determinante a suffragare l'ipotesi di L. Delisle quanto all'identità del traduttore. La studiosa fornisce in appendice l'edizione dei quattro 'exempla'.

3 La seconda sezione ha per titolo Tradition et traduction des classiques e si richiama agli importanti contributi forniti da G. Di Stefano nell'ambito della traduzione dal latino. GIOVANNA ANGELI (Christine de Pizan et la métamorphose, pp. 61-68) riflette sul tema della 'mutacion' che, seguendo il modello dell'Ovide Moralisé, compenetra profondamente le opere della poetessa dalla Mutacion de Fortune all'Avision Christine. ANNA MARIA BABBI (Jean de Meun traducteur de la 'Consolatio Philosophiae' de Boèce, pp. 69-77) affronta la traduzione dell'opera di Boezio da parte di Jean de Meun alla luce della sua esperienza di scrittore, mentre ANNE BERTHELOT (Une marqueterie d'auteurs antiques: l'ouverture du 'Roman de Perceforest', pp. 79-85) esamina il tema del rapporto tra scrittura romanzesca e legittimazione della narrazione nel prologo e nella lunga sequenza iniziale del Perceforest. Un aspetto particolare della ricezione di Plutarco nel Medioevo è l'oggetto del contributo di CHARLES BRUCKER (La pensée morale et politique de Plutarque dans un Miroir des princes latin du XII ${ }^{\mathrm{e}}$ siècle, pp. 87-99), che mostra attraverso precisi rinvii testuali la profonda influenza esercitata dalle opere di Plutarco, e particolarmente dalla lettera a Traiano, sul Policraticus di Jean de Salisbury, tradotto da Denis Foulechat per Carlo V. PIERRE DEMAROLle (Traductions et traducteurs chez La Croix du Maine, pp. 101-110) studia le traduzioni di opere classiche recensite nella bio-bibliografia di La Croix du Maine, mostrando non solo quali sono i classici più rappresentati, ma anche la logica che sottende le scelte effettuate dall'erudito cinquecentesco, frutto dell'epoca nella quale quest'ultimo compilò il suo repertorio. La traduzione come creazione originale, che deve da un lato competere con il prestigio del testo-fonte e dall'altro assecondare le esigenze del pubblico umanista erudito viene affrontata da LUDMILLA EVDOKIMOVA ( $L a$ traduction en vers des comédies de Térence dans l'édition d'Antoine Vérard: le choix du style et du destinataire, pp. 111-22), che studia la funzione estetica svolta da amplificazioni, costruzioni sintattiche, neologismi nel volgarizzamento di Terenzio pubblicato all'inizio del Cinquecento dall'editore parigino Antoine Vérard. MICHÈLE GOYENS (Évrart de Conty: traducteur, adaptateur et commentateur des 'Problèmes' d'Aristote, pp. 123-36) mostra che la traduzione dell'opera aristotelica realizzata da Évrart de Conty a partire dalla versione di Bartolomeo da Messina costituisce un esempio di equilibrio tra fedeltà e chiarezza, analizzando in particolare le amplificazioni, le modalità di inserzione delle glosse e gli spunti più personali del traduttore, che si fa talvolta critico del filosofo greco. VIRGINIE MINET-MAHY ('Si semoit sel en leu d'avaine' (OM, XII, v. 1031). De la traduction d'Ovide aux figures d'auteur: Ulysse et le bouclier d'Achille, pp. 137-54) esamina la figura di Ulisse nel celebre episodio della follia, mettendo in evidenza che la rappresentazione di 
questo personaggio fornita dall'Ovide Moralisé è fondamentale per la posterità, poiché esso diventa non solo il simbolo della clergie éclairée, ma anche un'immagine del clerc che ambisce a diventare consigliere dei potenti. MARTINE THIRY-STASSIN (Un traducteur fidèle. Quelques remarques sur la traduction de la 'Vita sancti Petri Thomae' de Philippe de Mézières, pp. 155-64) studia invece la traduzione di una parte del libro III dell'opera di Philippe de Mézières realizzata dal carmelitano Thomas de Lamborc alla fine del Quattrocento e conservata in tre manoscritti. Nel contributo di JEAN-JACQUES VINCENSINI (Un prologue inédit du roman de Jean d'Arras, 'Mélusine' ou 'La Noble Histoire de Lusignan'. Notes sur l'aristotélis-me moral et politique, pp. 165-82), il prologo contenuto nel ms Vienna, Österreichische Nationalbibliothek 2575 della Mélusine di Jean d'Arras viene trascritto ed analizzato soprattutto sulla base dei numerosi rinvii all'Etica Nicomachea di Aristotele. A Valerio Massimo e alla traduzione realizzata da Simon de Hesdin è dedicato lo studio di ALESSANDRO VITALE-BROVARONE (Notes sur la traduction de Valère Maxime par Simon de Hesdin, pp. 183-91), che chiude questa sezione sollevando alcuni problemi di datazione, statuto dei testimoni, metodo di lavoro del traduttore, pubblico a cui era destinato questo volgarizzamento, a cui G. Di Stefano ha dedicato pagine fondamentali.

Un altro campo di interesse centrale per il dedicatario della miscellanea è rappresentato dalle problematiche legate all'edizione critica dei testi in moyen français, che costituiscono l'argomento della terza sezione, intitolata L'Édition de textes: notion de corpus - notion de texte. Il primo contributo (MONICA BARSI, Le récit de Jacopo Tedaldi sur la prise de Constantinople: édition du manuscrit BNF, fonds français 6487, pp. 195-208) fornisce l'edizione, preceduta da uno studio della tradizione manoscritta, delle Informations sulla caduta di Bisanzio redatte nel 1453, probabilmente in italiano, dal mercante fiorentino Jacopo Tedaldi e conservateci in versione francese. MARIA COLOMBO Timelli (Les 'Principia grammaticalia' de Martin Morin, Rouen, s.d. [1498]. Introduction et édition, pp. 209-24) pubblica il primo manuale per l'insegnamento del latino apparso a stampa in Normandia; dopo aver inserito questo testo nell'ambito delle opere a carattere pedagogico e nell'insieme della produzione del libraio rouennese M. Morin, l'A. ne sottolinea l'interesse lessicografico, a causa dei numerosi neologismi, e documentario, per la conoscenza del ruolo del francese nell'insegnamento del latino. Segue l'edizione critica. Una delle due traduzioni in moyen français di un trattato profetico composto nel 1356 dal francescano Johannes de Rupescissa è analizzata da BARBARA FERRARI (Le 'Vade mecum in tribulatione' de Jean de Roquetaillade en moyen français, ms. BAV, Reg. lat. 1728, pp. 225-36), che prende in esame le tecniche di riscrittura del testo latino, notevolmente abbreviato, e la qualità di questo volgarizzamento. FRANÇoISE FÉRY-HUE ('Revelatio Esdrae' ou 'Prophéties d'Ezéchiel'. Éléments nouveaux pour le corpus latin et français des prophéties d'après le jour de Noël, pp. 237-52) studia le pronosticazioni che vanno sotto il nome di Revelatio Esdrae, utilizzate per conoscere il giorno della settimana in cui inizia l'anno, a cui viene aggiunta in epoca più tarda la profezia per il 25 dicembre. Tra i quindici testimoni di quest'ultima, l'A. individua quattro sezioni inedite, studiate e pubblicate nel presente lavoro. ANNA MARIA FINOLI (Saladin en deux cycles, pp. 253-64) ricostruisce la genesi del ciclo di Jehan d'Avennes così come si presenta nel ms BNF f.fr. 12.572 studiando la tradizione manoscritta e le tecniche narrative, oltre ai riferimenti interni. Dopo aver individuato come nucleo centrale La Fille du comte de Ponthieu, la studiosa si interroga sul significato della presenza del personaggio di Saladino in un ciclo che costituisce la storia di una famiglia. JEAN-FRANÇOIS KOSTA-THÉFAINE (Des règles 'en françois' pour bien se tenir à table, pp. 265-76) pubblica uno dei tre testi che 
costituiscono il corpus delle Contenances de Table, situandolo all'interno di una vasta tradizione e segnalandone le particolarità. $\mathrm{A}$ una questione puntuale riguardante $\mathrm{i}$ problemi di edizione è dedicato il contributo di PHILIPPE MÉNARD (Édition de texte et paléographie: le problème de transcription de ' $u$ ' et ' $n$ ', pp. 277-86), che analizza i casi controversi e problematici di scioglimento del titulus, raccomandando agli editori di testi il ricorso a nozioni di etimologia, filologia, fonetica storica per dirimere le questioni più spinose senza corrrere il rischio di fornire un'immagine 'fantasiosa' dell'ancien o del moyen français. Sulla base di due nuovi testimoni, più vicini al Catholicon, del noto glossario Aalma, BRIAN MERRILEES, WILLIAM EDWARDS e MICHELLE TROBERG (Vers une nouvelle édition du glossaire latin-français l' 'Aalma', pp. 287-92) effettuano sondaggi sulle voci della lettera $\mathrm{B}$ e concludono sulla necessità di un'edizione critica di questo testo, che sostituisca quella di M. Roques. Sempre il ritrovamento di un nuovo testimone, che si configura come il più antico, induce giovanNI PAlumbo (Le 'Roman d'Abladane' à la lumière du ms. Paris, BNF, Nouv. Acq. Fr. 18236, pp. 293-304) ad esporre alcune osservazioni preliminari all'edizione critica del Roman d'Abladane, racconto breve in prosa composto verso il 1260 e noto finora solo attraverso copie settecentesche. DANIELLE RÉGNIER-BOHLER (L'événement permanent ou la source recompensée. Le texte en question, pp. 305-12) esamina il motivo del cataclisma nel Livre du Chevalier de la Tour Landry, mostrando che esso si configura come la ripetizione di un modello biblico. TANIA VAN HEMELRYCK (Le 'Débat de Mars et du Cul'. Contrepoint érotico-guerrier et fricassée linguistique, pp. 313-23) analizza un breve testo in versi che fa parte del «sousbois touffu et herbeux des petits textes méconnus», conservato in un unico manoscritto quattrocentesco, di cui fornisce l'analisi e l'edizione critica.

Nella quarta sezione, dedicata a François Villon, JELle KoOPMANs ('Poste au cagnard': la locution, du théâtre à Villon; de Villon au théâtre: la locution: de Villon à Villon, pp. 327-38) si sofferma su una locuzione che possiede una connotazione argotique, di cui segnala attestazioni in Villon ed in due testi drammatici della fine del XV secolo; essa fornisce lo spunto per riflettere sui rapporti tra le ballate in jargon di Villon ed il teatro profano contemporaneo. È invece il motivo delle stagioni, e particolarmente l'inverno e l'estate rispettivamente nel Lais e nel Testament ad interessare BARBARA SARGENT-BAUR (L'hiver et l'été dans l'œuvre de François Villon, pp. 339-44).

6 La quinta sezione (Théâtre médiéval - la versification) rende omaggio al «pionnier des études de versification théâtrale médiévale dans la perspective la plus signifiante qui soit, c'est-à-dire le rapport que peuvent entretenir la forme avec le contenu discursif et le déroulement dramatique» (p. 423) e si apre con il saggio di JONATHAN BECK ('Les origines du théâtre classique en France': appropriations d'un mythe, de la Renaissance à nos jours, pp. 347-58), che pone questioni di carattere generale e critica la concezione storiografica secondo la quale l'anno 1550 costituirebbe un momento di svolta nella storia del teatro francese, mostrando come questo sia da considerarsi un mito creato dagli intellettuali cinquecenteschi. Esso nasconde invece una sostanziale continuità fra generi medievali sacri e profani e 'nuovi' generi classicheggianti. A uno di questi generi medievali è consacrato il contributo di ROSANNA BRUSEGAN (Encore sur le 'Jeu du Pèlerin': autoportrait d'Adam de la Halle?, pp. 359-66), che torna sulla questione dell'attribuzione del Jeu du Pelerin all'autore del Jeu de la Feuillee, dimostrando che il testo, scritto con intento editoriale più che drammaturgico, sarebbe da ascrivere ad un autore dell'entourage di Adam de la Halle, se non del poeta stesso. OLGA ANNA DUHL ('La Sotise a huit personnaiges': (un) drame (du) poétique, pp. 367-80) esamina la forma ibrida di questo testo a metà 
strada fra sotie e mystère, mostrando che in esso si realizza una sintesi tra generi diversi, che includono anche forme poetiche praticate dai Rhétoriqueurs, aventi un ruolo preciso nell'economia della pièce. CAROL HARVEY (Entre acte de foi et spectacle divertissant: les 'Miracles de Notre-Dame par personnages', pp. 381-90) studia tre dei quaranta miracles legati al culto mariano composti per la Confrérie de Saint-Eloi tra il 1339 ed il 1382; il tema centrale, quello della donna perseguitata ingiustamente, rivela che l'intento ludico in testi di questo genere convive con quello edificante. La rappresentazione parodica degli occupanti inglesi in testi teatrali composti durante la guerra dei Cent'Anni, nello studio di DENIS HÜE (Carrefour des langues: l'Anglais en France et l'Écossais en Italie, pp. 391-402), viene esaminata attraverso l'analisi di numerosi testi, a cui fa seguito l'edizione di tre ballate inserite nel manoscritto fr. 2206 del Jardin de Plaisance, dove le difficoltà interpretative pongono numerosi quesiti. Le questioni linguistiche sono al centro anche dello studio di TAKESHI MATSUMURA (Notes sur quelques régionalismes dans les farces françaises, pp. 403-408), che presenta alcuni complementi al lavoro di Y. Greub sui regionalismi nel Recueil des Farces pubblicato da A. Tissier, ed in quello di GABRIELLA PARUSSA, dedicato al linguaggio delle figure diaboliche in quattro mystères religiosi quattrocenteschi (Paroles de diables. Essai d'une typologie du discours diabolique dans les mystères religieux $d u X V^{e}$ siècle, pp. 409-22); la studiosa identifica i tratti che accomunano la 'parola diabolica' ed esamina il ruolo di questi personaggi nell'insieme della drammaturgia religiosa francese. Al Mystère de saint Martin di André de la Vigne ed alla sua strutturazione metrica è dedicato il contributo di CLAUDE THIRY ('Pour faire ... misteres bien limitez': observations sur la versification du 'Mystère de saint Martin' d'André de la Vigne, pp. 423-36), che prende in esame la strutturazione metrica della pièce e delle composizioni poetiche in essa inserite per suggerire, da un lato, lo stretto rapporto di questo testo con l'Instructif de seconde rhétorique e, dall'altro, la possibile influenza del Mystère du siège d'Orléans su questo testo composto, apparentemente, in un lasso di tempo molto breve.

7 Nella sesta sezione (La Réception de Boccace en France) i contributi si articolano intorno ad un tema che ha occupato a lungo G. Di Stefano, la cui monumentale e rigorosissima edizione del Decameron in francese tradotto da L. de Premierfait resta un punto di riferimento importante non solo per chi si occupa della fortuna del poeta italiano in Francia, ma anche per tutti coloro che si cimentano con l'edizione critica di testi in moyen français. Lo studio d'apertura è dovuto ad un altro illustre studioso di Boccaccio in Francia: GABRIEL BIANCIOTTO, editore della traduzione francese del Filostrato, si dedica qui (Les manuscrits du 'Livre de Thezeo', pp. 439-56) al Livre de Thezeo, adattamento in prosa del Teseida, ancora in gran parte inedito, studiando i rapporti fra i quattro testimoni della versione più completa e le caratteristiche del testo italiano di partenza. Segue poi lo studio di MARC-RENÉ JUNG (Une adaptation des 'Genealogie deorum' de Boccace dans une chronique française, pp. 457-66) che analizza il libro IV di una cronaca francese quattrocentesca conservata in cinque testimoni, mostrando l'influenza del modello boccacciano sulla sezione consacrata ai falsi dei e fornendo l'edizione di alcune sezioni particolarmente significative di questa parte del testo. L'influenza delle Genealogie boccacciane, ma anche dei Triumphi di Petrarca sul Roman du Cuer d'amours espris viene studiata da JeAN-Claude müHLETHALER (De la disparition de Didon dans le 'Roman du Cuer d'amour espris': René d'Anjou entre Boccace et Pétrarque ou la difficile récupération d'Enée en France au XV siècle, pp. 467-83) soprattutto in relazione alla figura di Enea, che figura nel romanzo di René d'Anjou fra gli antenati celebri e che subisce nel testo un processo 
di riabilitazione, almeno in parte parallelo a quello realizzato in altre opere coeve. Sempre le Genealogie e l'influenza esercitata da questo testo sugli autori quattrocenteschi sono alla base del lavoro di ANNE SCHOYSMAN (Jean Miélot, Jean Boccace et les généalogies. Notes sur le ms BNF, f.fr. 17001, pp. 483-91), che esamina un breve testo relativo alle norme per la redazione delle genealogie composto da Jean Miélot e contenuto in un codice della Bibliothèque Nationale de France; questo documento, già studiato qualche anno fa da Gianni Mombello, viene analizzato come esempio dello stretto rapporto che lega poesia e storiografia nella produzione di Miélot, per il quale "la poésie est une forme de l'historiographie, dont fait partie intégrante la généalogie" (p. 486). Torna sull'influenza esercitata dal Decameron sul Livre de la Cité des Dames di Christine de Pizan il contributo di ANNA SLERCA (Le 'Livre de la Cité des Dames' de Christine de Pizan, le 'Décaméron' et une guirlande de pervenches, pp. 491-500), che segnala un nuovo probabile influsso di Boccaccio sull'opera della poetessa francese, nella quale si ravvisano analogie con le novelle 6 e 7 della decima giornata. ELINA SUOMELA (Les pronoms d'adresse dans le 'Décaméron' et la traduction de Laurent de Premierfait, pp. 501-10) adotta un'interessante prospettiva linguistica per cercare di chiarire alcuni dei numerosi interrogativi suscitati dalla traduzione francese del Decameron a proposito del metodo adottato dal traduttore, il quale, come è noto, lavorò a partire da un intermediario latino non conservato; la studiosa si concentra sul tutoiement e vouvoiement, comparando il sistema del testo italiano con quello adottato nella traduzione francese ed osservando che, malgrado le interferenze che la traduzione latina avrebbe potuto comportare e le differenze tra il funzionamento dell'italiano e del francese nell'uso dei pronomi 'tu' e 'voi', il testo di Laurent de Premierfait riproduce esattamente lo schema dell'italiano.

8 L'ultima sezione di questo volume ha come filo conduttore Les Locutions, sulla scia dell'imponente dizionario compilato da G. Di Stefano ormai più di dieci anni fa. GIOVANNA BELLATI (Un phénomène de 'cristallisation' dans la traduction des 'Rerum vulgarium fragmenta' de Vasquin Philieul, pp. 513-26) studia alcuni stilemi caratteristici nella traduzione del Canzoniere realizzata a metà Cinquecento da Vasquin Philieul, mostrando che il traduttore seppe assimilare perfettamente il linguaggio poetico petrarchiano, immergendosi a tal punto nel testo italiano da poterne usare autonomamente immagini e figure di stile. CLAUDIO GALDERISI (Les incipits des poèmes de concours auréliens: du refrain aux marges locutionnelles, pp. 527-39) analizza il funzionamento poetico degli incipitritornelli nei componimenti realizzati da Charles d'Orléans per i concorsi di Blois, mostrando quanto tale funzionamento sia assimilabile a quello delle locuzioni e proponendo, su questa base, una classificazione dei componimenti contenuti nelle tre raccolte collettive del circolo orbitante intorno al principe-poeta. SYLVIE LEFÈVRE (Salade et jambon. Deux italianismes chez Antoine de La Sale ou petites histoires de langue et de bouche, pp. 539-48) propone alcune variazioni sulla storia di due italianismi utilizzati da Antoine de La Sale ed introdotti, almeno in uno dei due casi, attraverso la traduzione francese del Decameron. MICHEL OLSEN (Polyphonie et monophonie au Moyen Âge. À propos du style indirect libre, pp. 549-56) analizza la presenza del discorso indiretto libero nelle opere in moyen français e le difficoltà per identificarlo in testi dove la punteggiatura fa difetto. GILBERT OUY e CHRISTINe M. RENo (Les 'Proverbes moraux' de Christine de Pizan. Une nouvelle édition critique et quelques problèmes, pp. 557-72) forniscono una nuova edizione critica dei Proverbes moraux di Christine de Pizan sulla base di un nuovo testimone, il ms BNF f.fr. 24864, che presenta varie lezioni interessanti; gli studiosi formulano anche 
alcune ipotesi sulla natura del manoscritto contraddistinto dagli editori precedenti con la lettera B e sulla composizione del testo. GILLES ROQUES ("Bruit" en français médiéval. Étude sémasiologique et onomasiologique, pp. 573-82) realizza uno studio onomasiologico in chiave diacronica del sostantivo bruit e dei suoi sinonimi, mentre DAVID TROTTER ( $L e$ problème de l'identification des locutions dans une langue morte: l'exemple de l"Anglo-Norman Dictionary', pp. 583-92) esamina lo spinoso problema dell'identificazione delle locuzioni, mostrando attraverso esemplificazioni tratte dalla versione elettronica dell' AngloNorman Dictionary che l'apporto dell'informatica e dei criteri quantitativi si affianca ormai efficacemente agli strumenti critici più tradizionali per gli studi nell'ambito della lessicografia. MARIA TERESA ZANOLA (Argent et monnaies au XV siècle entre réalité historique et métaphorisation, pp. 593-99) esamina, a partire dal Dictionnaire des locutions en moyen français di G. Di Stefano, le locuzioni concernenti la moneta e concentrandosi sul loro uso metaforico nel Triparty en la science des nombres, trattato d'algebra composto nel 1484. Chiude il volume lo studio di OTTAVIO LURATI (Nomi di luogo e architettura medievale (bellica e idraulica), pp. 601-608) che analizza una serie di toponimi appartenenti ad un nucleo semantico omogeneo riconducibile al lessico dell'architettura, concentrandosi in particolare su quelli derivati dai sostantivi 'trabucco', 'briccola', 'cassero', 'ribellino' e fornendo alcune riflessioni metodologiche. 